\title{
On the origin of the $\gamma$-ray emission from the flaring blazar PKS 1222+216
}

\author{
F. Tavecchio ${ }^{1}$, J. Becerra-Gonzalez ${ }^{2,3}$, G. Ghisellini ${ }^{1}$, A. Stamerra ${ }^{4}$, G. Bonnoli ${ }^{1}$, L. Foschini ${ }^{1}$, and L. Maraschi ${ }^{5}$ \\ 1 INAF - Osservatorio Astronomico di Brera, via E. Bianchi 46, 23807 Merate (LC), Italy \\ e-mail: fabrizio.tavecchio@brera.inaf.it \\ 2 Instituto de Astrofisica de Canarias (IAC), 38200 La Laguna, Tenerife, Spain \\ 3 Depto. Astrofisica, Universidad de La Laguna (ULL), 38206 La Laguna, Tenerife, Spain \\ 4 Dipartimento di Fisica, Università di Siena, and INFN Pisa, 53100 Siena, Italy \\ 5 INAF - Osservatorio Astronomico di Brera, via Brera 28, 20121 Milano, Italy
}

Received 6 May 2011 / Accepted 4 August 2011

\section{ABSTRACT}

\begin{abstract}
The flat-spectrum radio quasar PKS $1222+216(4 \mathrm{C}+21.35, z=0.432)$ was detected in the very high energy $\gamma$-ray band by MAGIC during a highly active $\gamma$-ray phase following an alert by the Large Area Telescope (LAT) onboard Fermi. Its relatively hard spectrum (70-400 GeV photon index $\Gamma=2.7 \pm 0.3$ ) without a cut off, together with its observed variability on a timescale of $\sim 10$ min challenges standard emission models. In particular, if the emission originates in a portion of the relativistic jet located inside the broad line region (BLR), severe absorption of $\gamma$ rays above a few tens of $\mathrm{GeV}$ is expected to be caused by the $\gamma \gamma \rightarrow \mathrm{e}^{ \pm}$process. These observations therefore imply that there is a very compact $\left(R_{\mathrm{b}} \sim 5 \times 10^{14} \mathrm{~cm}\right)$ and rapidly moving blob located far beyond the BLR radius (to avoid the gamma-ray absorption through pair production) that is responsible for the rapidly varying high energy flux. However, the long-term (day-week) coherent evolution of the GeV flux recorded by LAT indicates that there could also be a substantial contribution from another, larger emission region. We model the spectral energy distribution of PKS 1222+216 during the epoch of the MAGIC detection assuming three different scenarios, namely: (1) a one-zone model considering only the emission from a compact blob outside the BLR; (2) a two-zone model consisting of a compact blob plus an emitting region encompassing the whole jet cross-section located outside the BLR; and (3) a two-zone model with the jet emitting region inside the BLR. In all cases we find that the high-energy emission from the compact blob is dominated by the inverse Compton scattering of the infrared thermal radiation of the dusty torus. Furthermore, both regions are matter-dominated, with the Poynting flux providing a negligible contribution to the total jet power. These results do not support models in which the compact blob is the result of reconnection events inside the jet or "needles" of highenergy electrons accelerated close to the BH. The observational framework and our radiative models might instead be compatible with scenarios in which the jet is re-collimated and focussed at large distances from the central $\mathrm{BH}$.
\end{abstract}

Key words. radiation mechanisms: non-thermal - $\gamma$-rays: galaxies - quasars: individual: $4 \mathrm{C}+21.35$

\section{Introduction}

Blazars emitting $\gamma$ rays at very high energy (VHE, $E>$ $100 \mathrm{GeV}$ ) are still a minority of the whole population, but their numbers are steadily increasing thanks to the new generation of sensitive Cherenkov arrays (e.g. De Angelis et al. 2008; Hinton \& Hofmann 2009). The majority of them are BL Lac objects, in particular those with the synchrotron bump peaking in the UV/X-ray band (highly peaked BL Lac, HBLs, Padovani \& Giommi 1995). The characteristic lack of significant thermal components in their spectra suggests that the jet propagates in a relatively "clean" environment. It is therefore widely believed that the high energy component is due to the inverse Compton (IC) scattering of the synchrotron photons themselves (synchrotron self-Compton model, SSC, e.g. Maraschi et al. 1992; Bloom \& Marscher 1996; Tavecchio et al. 1998).

The detection in the VHE band of a few blazars belonging to the class of flat spectrum radio quasar (FSRQs) is particularly interesting. The broad emission lines observed in their optical-UV spectra, typical of quasars, are thought to originate in the socalled broad line region $(\mathrm{BLR})$, where dense $\left(n \simeq 10^{9-10} \mathrm{~cm}^{-3}\right)$ clouds of gas orbiting at typical velocities of a few thousands of $\mathrm{km} \mathrm{s}^{-1}$ at distances $R_{\mathrm{BLR}} \approx 10^{17-18} \mathrm{~cm}$ from the central black hole (BH) (e.g. Kaspi et al. 2007; Bentz et al. 2009) are photoionized by the UV continuum of the accretion disk. In this case inside the BLR, i.e. at distances $d<R_{\mathrm{BLR}}$, the environment is rather rich in optical-UV photons, which are ideal targets for the IC scattering with the relativistic electrons in the jet. If the high-energy hump of FSRQs is produce primarily by this mechanism (external Compton scenarios, EC, e.g., Sikora et al. 1994; Ghisellini et al. 1998; Dermer et al. 2009), a pronounced softening of the spectrum is expected above a few tens of $\mathrm{GeV}$ owing to the low scattering efficiency (e.g. Tavecchio \& Ghisellini 2008; Ghisellini \& Tavecchio 2009). More importantly, the dense radiation field makes the environment strongly opaque to $\gamma$ rays above a few tens of $\mathrm{GeV}$ (e.g. Liu \& Bai 2006; Tavecchio \& Mazin 2009; Poutanen \& Stern 2010; but see Stern $\&$ Poutanen 2011). The combination of these two mechanisms, which are effective above similar thresholds, makes the detection of VHE emission by FSRQs rather surprising. Even if the BLR is assumed to be "flat", as suggested in the past (e.g. Shields 1978) and by recent studies (e.g. Jarvis \& McLure 2006; Decarli et al. 2011), the absorption cannot be avoided (Tavecchio et al., in prep.).

The prototypical FSRQ 3C 279 has been detected by MAGIC on two occasions, a few days after optical flares 
(Albert et al. 2008; Aleksic et al. 2011a), with an extremely soft spectrum and a flux that was variable on a timescale of a day. Another FSRQ, PKS 1510-089, was detected at VHE by H.E.S.S. (Wagner \& Behera 2010). The recent detection of the FSRQ PKS 1222+216 (also known as 4C+21.35, $z=0.431$, Osterbrock \& Pogge 1987) by MAGIC (Aleksic et al. 2011b) is particularly challenging. The VHE spectrum smoothly connects to the LAT spectrum (Tanaka et al. 2011) and is well-described by a rather hard power-law, which is difficult to reconcile with the expected effects of absorption and decreased scattering efficiency discussed above. Moreover, the rapidly varying VHE flux, with a doubling in flux within about $10 \mathrm{~min}$, constrains the emitting-region size to be $R<c t_{\mathrm{var}}(1+z) \delta \simeq 2.5 \times 10^{14}(\delta / 10) \mathrm{cm}$ ( $\delta$ is the relativistic Doppler factor). If these dimensions are related to the jet cross-sectional radius, as in the standard one-zone scenarios, this implies that the emission region is located close to a black hole, hence deeply inside the opaque BLR radiation field.

As already proposed in Aleksic et al. (2011b), a possible solution to the problem is to assume that the region producing VHE $\gamma$ rays lies beyond the BLR, at distances $d>R_{\mathrm{BLR}}$. At these distances, the external radiation field is likely dominated by the thermal radiation of the dusty torus reprocessing the disk radiation (e.g. Błażejowski et al. 2000) and both the opacity and the reduced scattering efficiency problems are relaxed. However, even for extremely small values of the jet collimation angle ( $<1 \mathrm{deg})$, at these distances we expect the jet cross-sectional radius to far exceed the limit imposed by the rapid variability (see also Tavecchio et al. 2010; Foschini et al. 2011). Hence, we have to assume either (as proposed by Ghisellini \& Tavecchio 2008; Giannios et al. 2009; Marscher \& Jorstad 2010) the existence of very compact emission regions embedded in the flow (as already done to explain the ultra-fast variability events observed in the two BL Lacs PKS 2155-304, Aharonian et al. 2007; and Mkn 501, Albert et al. 2007) or strong recollimation and focusing of the flow (e.g. Stawarz et al. 2006; Bromberg \& Levinson 2009; Nalewajko \& Sikora 2009).

We note that the problems posed by the interpretation of this observational evidence for blazars have strict analogies with the issues raised by the unexpected recent discovery of rapid ( day) variability of the $\gamma$-ray emission of the Crab nebula by AGILE (Tavani et al. 2011) and Fermi (Abdo et al. 2011). As in blazars, to account for these short timescales it seems necessary to admit the existence of very compact knots inside the large-scale relativistic wind from the pulsar (Vittorini et al. 2011; Bednarek \& Idek 2011), whose non-thermal emission is possibly boosted by relativistic effects (Komissarov \& Lyutikov 2011). It is therefore tempting to assume that similar physical processes, possibly related to plasma instabilities in relativistic flows, operate and shape the emission in rather different $\gamma$-ray sources.

In this paper, we consider the problems posed by the observation of PKS $1222+216$ focusing whether standard leptonic models can reproduce the observed spectral energy distribution (SED) and variability and, in particular, whether a one-zone model is still a viable solution. To achieve this aim we consider whether a single, extremely compact emitting-region (a "blob") located beyond the BLR can account for the spectral and variability observed properties, concluding that, on the basis of the results of the SED modeling and the jet power requested by the model, this solution could be viable. Alternatively, we study a scenario based on two emitting regions, a "standard" emission region encompassing the whole jet cross-section plus a compact "blob" responsible for the rapidly varying high-energy emission detected by MAGIC. This possibility is in part motivated by the observed long-term smooth evolution shown by the LAT light curve, which is difficult to explain if the emission originates from uncorrelated, rapidly-evolving blobs. Moreover, we show that this case is also energetically less demanding than the single blob model. This two-zone scenario resembles that already discussed by Ghisellini \& Tavecchio (2008) for the case of PKS 2155-304. However, the existence of an intense external radiation field makes the situation somewhat different, because the radiative interplay between the two regions is less important than the EC emission with the IR photons (see Appendix B).

We construct the SED using nearly simultaneous Swift (UVOT and XRT), Fermi/LAT, and MAGIC data (Sect. 2). We then apply the models to reproduce the SED (Sect. 3). Finally, we discuss the results (Sect. 4). We adopt the cosmological parameters $H_{0}=70 \mathrm{~km} \mathrm{~s}^{-1} \mathrm{Mpc}^{-1}, \Omega_{\Lambda}=0.7$, and $\Omega_{\mathrm{M}}=0.3$.

\section{The spectral energy distribution}

MAGIC detected PKS $1222+216$ in a short ( $30 \mathrm{~min})$ observation on 2010 June 17 (MJD 55364.9), during a period of exceptional GeV activity (Tanaka et al. 2011). In Fig. 1, we present data taken from Aleksic et al. (2011b) corrected for absorption caused by the interaction with the extragalactic background light modeled as in Dominguez et al. (2011). Aleksic et al. (2011b) also presented LAT data-points and the corresponding spectral "bow-tie" obtained for a short time of $2.5 \mathrm{~h}$ encompassing the MAGIC observation. For comparison, the black thick solid line (from Tanaka et al. 2011) shows the LAT spectrum during quiescence.

In 2010 May-June Swift observed PKS 1222+216 several times, during which the source experienced high activity. Although no pointings are exactly simultaneous with the MAGIC observation, an observation was made a few days after, on June 20, when the average daily LAT flux was similar to that on June 17 (see the LAT light curve in Tanaka et al. 2011).

The XRT (total exposure time of $4.5 \mathrm{ks}$ ) data obtained during this observation (in PC mode) were analysed using the xrtpipeline task considering grade $0-12$ events and using v6.10 version of the HEASOFT package and the calibration files caldb 9/2/2011. As commonly observed in FSRQs, the XRT spectrum (red) is relatively hard with a photon index $\Gamma=1.60 \pm$ 0.13 (the fit gives $\chi^{2}=9.25$ for 11 d.o.f.). The $0.3-10 \mathrm{keV}$ flux is $F_{\mathrm{X}}=5.5 \times 10^{-12} \mathrm{erg} \mathrm{cm}^{-2} \mathrm{~s}^{-1}$. For comparison, we also analyzed the data corresponding to a previous observation on May 29. The flux was then a factor $\approx 1.7$ higher, $F_{X}=8.3 \times 10^{-12} \mathrm{erg} \mathrm{cm}^{-2} \mathrm{~s}^{-1}$, and the spectrum (cyan) was softer than on June 20 , with $\Gamma=$ $2.12 \pm 0.14\left(\chi^{2}=10.6\right.$ for 11 d.o.f. $)$. We to note that while X-ray spectra of powerful FSRQs are generally hard, $\Gamma \leqslant 1.5$ (e.g. Kubo et al. 1998; Tavecchio et al. 2000, 2002), soft X-ray slopes (indicating a dominant contribution of the synchrotron or SSC component in this band) are occasionally also shown by the other two TeV detected FSRQs, 3C 279 (e.g. Aleksic et al. 2011a) and 1510-089 (e.g. Tavecchio et al. 2000).

The UVOT data-points (obtained with the standard procedure, e.g. Bonnoli et al. 2011) reveal a rather hard optical-UV spectrum that is indicative of direct thermal emission from the accretion disc (see e.g. Ghisellini et al. 2009a,b, 2011, for a discussion). To investigate this point, we also consider SDSS data (taken on January 2008). To directly compare the UVOT and SDSS photometry, we convert SDSS ugriz magnitudes into the standard Johnson system using the formulae provided by Chonis \& Gaskell (2008). Using standard zero-points, we finally convert magnitudes into fluxes (magenta open squares). The SDSS data confirm the presence of a rather hard continuum. However, for 


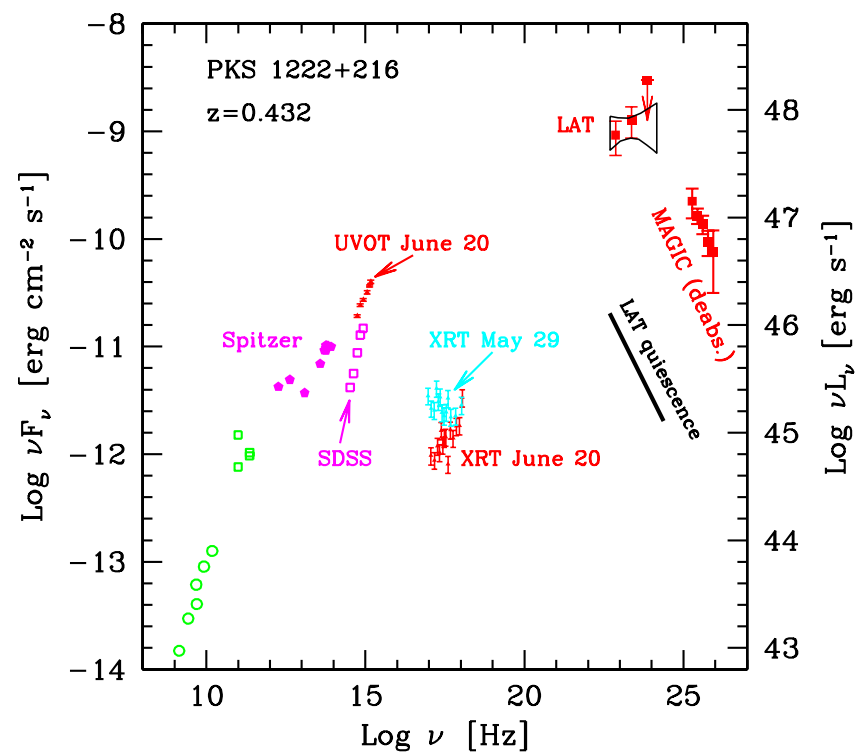

Fig. 1. Spectral energy distribution of PKS 1222+216 close to the epoch of the MAGIC detection (2010 June 17). Red points at optical-UV and X-ray frequencies are from a Swift observation of June 20. For comparison, cyan data-points show the X-ray spectrum two weeks before, on May 29 (see text). Fermi/LAT (red squares and "bow tie") and MAGIC data (corrected for absorption by the EBL using the model of Dominguez et al. 2011) are taken from Aleksic et al. (2011b). The thick black solid line shows the LAT spectrum in quiescence (from Tanaka et al. 2011). Magenta open squares are SDSS photometric points. Magenta filled pentagons are IR data from Malmrose et al. (2011). Green points report historical data (from NED, circles, and Tornikoski et al. 1996, squares).

the overlapping filters $(U, B$, and $V)$ there is a systematic difference of about $0.8 \mathrm{mag}$ between UVOT and SDSS, translating into UVOT fluxes that are higher by a factor of two, as clearly visible in the SEDs. This difference could represent an increase in the accretion luminosity between the SDSS (Jan. 2008) and the UVOT (June 2010) observations, that is possibly related to the high activity in $\gamma$ rays.

Malmrose et al. (2011) reported on Spitzer observations in the IR band for four sources, including PKS 1222+216. The IR data points (filled magenta pentagons in Fig. 3) track a bump around $3 \mu \mathrm{m}$ that is fitted well by a black body of temperature $T=1200 \mathrm{~K}$ and is clearly related to the thermal emission from the putative dusty torus. Finally, we also add historical radio (from NED) and millimeter (Tornikoski et al. 1996) data (green open circles and open squares, respectively).

\section{Modeling the SED}

\subsection{Observational findings and problems}

In modeling the observed SED, we are constrained/guided by the following observational findings: 1) the MAGIC VHE spectrum $(70-400 \mathrm{GeV})$ is well-described by a hard power-law, with photon index (after correction for absorption by the interaction with the extragalactic background light) of $2.7 \pm 0.3$, and a cutoff for energies lower than $130 \mathrm{GeV}$ is excluded. This spectrum smoothly connects to the LAT spectrum close to the MAGIC detection (Tanaka et al. 2011), strongly suggesting that both the high-energy and VHE emission belong to a unique spectral component, originating in the same region; 2) the MAGIC lightcurve shows a significative increase in the flux during the $30 \mathrm{~min}$ observation, with a doubling time of about $t_{\mathrm{var}} \simeq 10 \mathrm{~min}$. The causality relation $R<c t_{\mathrm{var}}(1+z) \delta$ allows us to constrain the size of the emitting region to be $R<2.5 \times 10^{14}(\delta / 10) \mathrm{cm}$ for typical values of the Doppler factor $\delta=10 ; 3$ ) the LAT long-term lightcurve (Tanaka et al. 2011) is characterized by periods of quiescence and smooth, long-lasting (about one week) flares. The MAGIC detection coincides with the raising part of a flare lasting for approximately three days. The $\gamma$-ray LAT flux $\left(F_{>100 \mathrm{MeV}} \sim\right.$ $\left.6.5 \times 10^{-6} \mathrm{ph} \mathrm{cm}^{-2} \mathrm{~s}^{-1}\right)$ was about half that recorded at the maximum of the flare $\left(F_{>100 \mathrm{MeV}} \sim 13.5 \times 10^{-6} \mathrm{ph} \mathrm{cm}^{-2} \mathrm{~s}^{-1}\right)$, which was reached the day after the MAGIC detection.

Standard one-zone models for FSRQ generally assume that a single region in the jet, with a size comparable to that of the jet cross-sectional radius, is responsible for the emission from IR to $\mathrm{GeV}$ frequencies. The location of this region is generally assumed to be inside the BLR (e.g. Dermer et al. 2009; Ghisellini \& Tavecchio 2009), but scenarios considering regions beyond it have been discussed (e.g., Sikora et al. 2008; Marscher et al. 2008).

The observational findings listed above already pose some problems for this scenario. Points 1) and 2) imply that the entire $\mathrm{MeV}-\mathrm{GeV}$ and VHE emission component at the epoch of the MAGIC detection was produced in a very compact emission region outside the BLR, to minimize the expected severe absorption above $10 \mathrm{GeV}$ (but see Stern \& Poutanen 2011). In the framework of one-zone models, a first possibility is that the entire $\gamma$-ray activity is due to the cumulative emission of very compact, uncorrelated traveling regions (resulting from, e.g. internal shocks, Spada et al. 2001). However, in this case the expected erratic behavior disagrees with the smooth long-term evolution shown by LAT. One way to reconcile this scenario with point 3 ) might be to assume the existence of a very compact and stationary region: this would allow rapid variations in the flux and, at the same time, the long-term modulation of the jet power would account for the smooth and coherent evolution. As an alternative, we could envision the existence of two emitting regions, a large region responsible for the long-term evolution visible in the LAT band and an extremely compact region accounting for the fast variations.

Motivated by the arguments above, we present below three different scenarios for the VHE flare of PKS 1222+216 (see Fig. 2). In the first case (A), we assume that the entire SED is produced by a single compact blob outside the BLR. In the other two cases, we consider a two-zone model with the large region located outside $(\mathrm{B})$ or inside $(\mathrm{C})$ the BLR. For consistency with the scenario sketched above, in cases B and C we consider that the large region might contribute substantially to (even if not dominate) the LAT band also at the epoch of the MAGIC detection.

\subsection{Model setup}

A sketch of the assumed geometry is shown in Fig. 2. In all cases, a central BH is surrounded by an accretion disk, whose radiation, of luminosity $L_{\mathrm{d}}$, photoionizes the BLR, modeled as a spherical shell located at distance $R_{\mathrm{BLR}}$ from the $\mathrm{BH}$. Following Ghisellini \& Tavecchio (2009), we set $R_{\mathrm{BLR}}=10^{17} L_{\mathrm{d}, 45}^{0.5} \mathrm{~cm}$. This relation provides a good approximation of the most recent results of the reverberation mapping studies (e.g. Kaspi et al. 2007; Bntz et al. 2009). We assume that the BLR clouds intercept and reprocess (mainly into emission lines) a fraction $\xi_{\text {BLR }}$ of $L_{\mathrm{d}}$. As discussed in Tavecchio \& Ghisellini (2008), a rather good approximation for the spectrum of the BLR radiation field as seen in the comoving frame is a black body with the peak at $v_{\mathrm{BLR}}^{\prime} \approx 3 \times 10^{15} \Gamma \mathrm{Hz}$. 

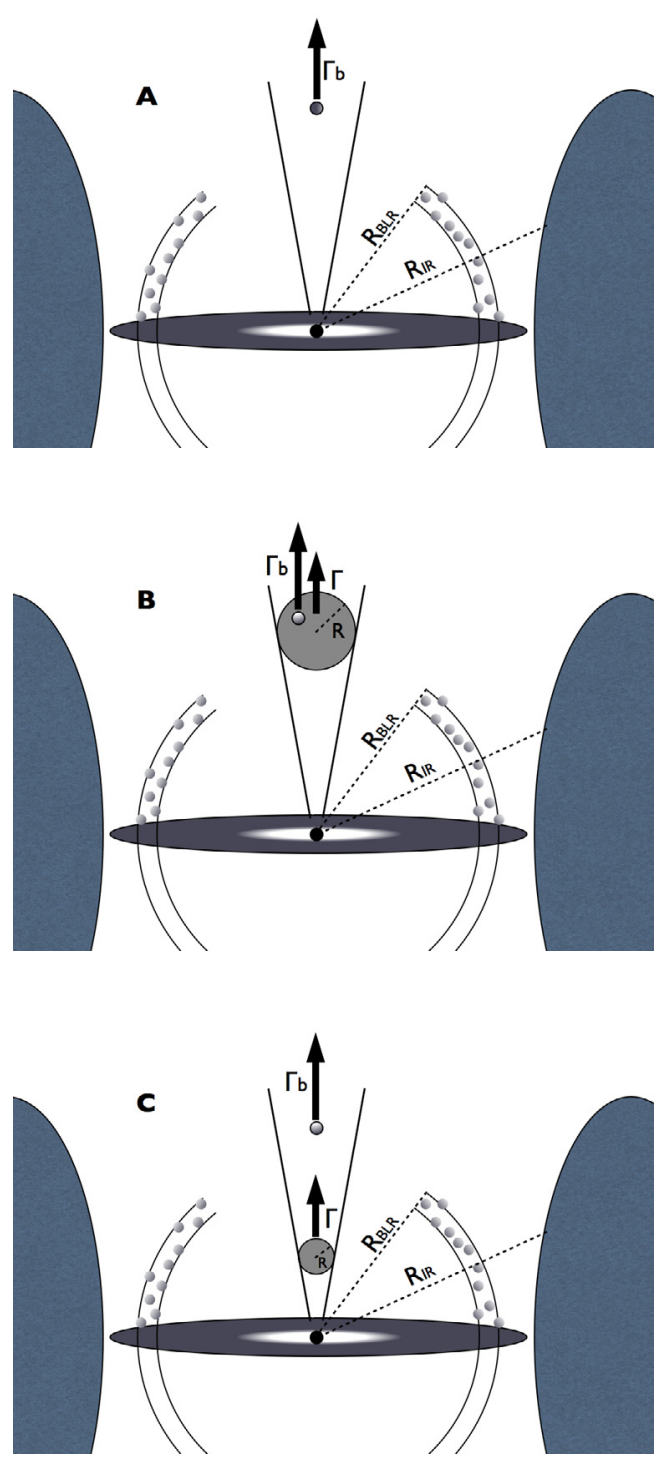

Fig. 2. Sketch of the geometrical arrangement assumed in the model (not to scale). We consider a spherical BLR with radius $R_{\mathrm{BLR}}$ and a dusty torus at $R_{\mathrm{IR}}$. In all cases, we consider the emission from a small compact "blob" of radius $R_{\mathrm{b}}$ moving with Lorentz factor $\Gamma_{\mathrm{b}}$. While in case A we assume that the blob is responsible for the entire SED, in cases B and $\mathrm{C}$, we also consider the emission from a "standard" spherical emission of radius $R$ equal to the cross-sectional size of a conical jet with semiaperture angle $\phi$, moving with bulk Lorentz factor $\Gamma$ located outside (B) or inside (C) the BLR. Each region is characterized by different values of the physical parameters, such as the magnetic field, electron density, and energies. See text for more details.

Since the UVOT data-points probably trace the direct disk emission, we fix $L_{\mathrm{d}}$ by reproducing the UVOT fluxes with a black body. Assuming that the peak corresponds to the UVOT filter at the highest frequency (UVW2), a lower limit to the disk luminosity is $L_{\mathrm{d}}=5 \times 10^{46} \mathrm{erg} \mathrm{s}^{-1}$ (Fig. 3, black short dashed line). This luminosity is exactly ten times higher than that estimated by Tanaka et al. (2011) using the BLR total luminosity (and assuming $\xi_{\mathrm{BLR}} \sim 0.1$ ), in turn inferred from the luminosity of the $\mathrm{H} \beta$ line in the SDSS spectrum (Fan et al. 2006). Considering the difference between the flux at the epoch of the SDSS (Jan. 2008) and UVOT measurements discussed in Sect. 2 we conclude that the discrepancy between the two

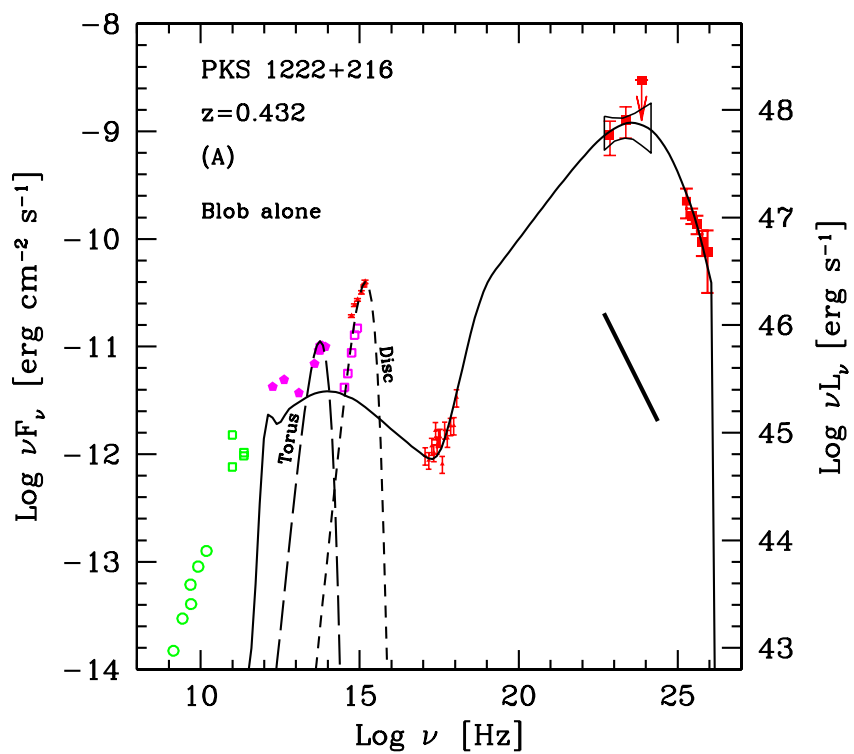

Fig. 3. As in Fig. 1 but with the model considering only a compact emission region located beyond the BLR (see text for details). Black short and long dashed curve show the assumed emission from the accretion disk and dusty torus. For clarity, the XRT spectrum of May 29 is not presented.

values of the derived BLR luminosity is smaller by a factor of $\approx 2$. A difference of a factor of 5 between the two estimates could be explained by assuming that $\xi_{\mathrm{BLR}} \sim 0.02$. Setting $L_{\mathrm{d}}=5 \times 10^{46} \mathrm{erg} \mathrm{s}^{-1}$, we have $R_{\mathrm{BLR}}=7 \times 10^{17} \mathrm{~cm}$.

Outside the BLR, a dusty torus intercepts and re-emits part of the central disk emission. The radiation field of the torus is modeled as a black body with temperature $T_{\mathrm{IR}}=1.2 \times 10^{3} \mathrm{~K}$ and total luminosity $L_{\mathrm{IR}}=10^{46} \mathrm{erg} \mathrm{s}^{-1}$ (black long dashed line in Fig. 3) filling a volume that, for simplicity, is approximated as a spherical shell with radius $R_{\mathrm{IR}}=7 \times 10^{18} \mathrm{~cm}$.

In all cases, we model the compact emission region as a sphere with radius $R_{\mathrm{b}}$ (the subscript "b" marks all the physical quantities related to the blob), moving with bulk Lorentz factor $\Gamma_{\mathrm{b}}$, filled with uniform and tangled magnetic field $B_{\mathrm{b}}$. We assume that relativistic electrons follow a smoothed broken powerlaw energy distribution of normalization $K$ and slopes $n_{1}$ and $n_{2}$ below and above the break at the energy $\gamma_{\mathrm{p}} m_{\mathrm{e}} c^{2}$.

We assume that a conical jet propagates from the $\mathrm{BH}$ vicinity, with semi-aperture angle $\phi=0.1 \mathrm{rad}$. A spherical region at distance $d$ and radius $R=\phi d$ moving along the jet with bulk Lorentz factor $\Gamma$, carries a tangled magnetic field (of uniform intensity $B$ ) and relativistic electrons with a distribution of the same functional form as in the case of the blob.

In case (A), we consider only the emission from the compact blob. In cases (B) and (C), we consider the emission from both regions.

Both regions emit synchrotron and IC radiation. For the blob and the jet components, we consider as seed photons for the IC scattering the locally-produced synchrotron photons (synchrotron self-Compton mechanism, SSC), the thermal emission from the dusty torus (EC/IR) and, in case (B), the synchrotron photons produced in the other region of the jet (which we refer to them as the EC/J and EC/b component)s. In case $(C)$ for the large region, we also consider the photons reprocessed from the BLR (EC/BLR). The calculations were performed adapting the code described in detail in Maraschi \& Tavecchio (2003). 

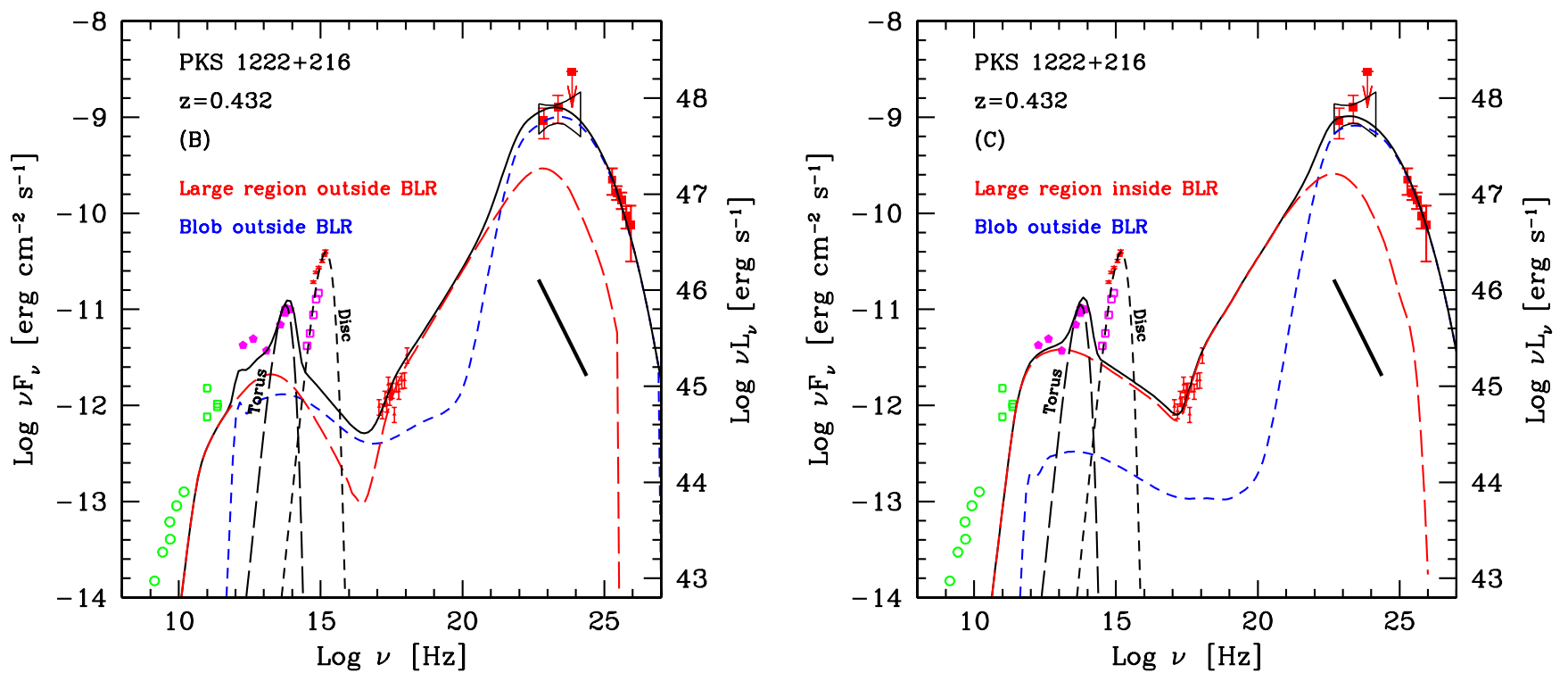

Fig. 4. As in Fig. 3 but with the model corresponding to emission from the compact region and the standard jet. The blue short dashed line shows the emission from the compact region, while the red long dashed line reports the emission from the large region of the jet. The solid black line is the sum of the two. The left panel shows the case in which both emission regions are located outside the BLR (case B); the right panel represents the case in which the large region is within the BLR (case C).

Since by construction the contribution of the large region is always negligible above $20-30 \mathrm{GeV}$, for simplicity we neglect absorption for this component when considering the region inside the BLR. For the blob, we expect to detect a very weak absorption produced the IR radiation field of the torus, that is significant at energies higher than a few $\mathrm{TeV}$. We also neglect this effect in the spectra shown in Figs. 3 and 4.

\subsection{Results}

We first consider (case A) the possibility that the very compact blob emitting $\mathrm{TeV}$ photons is responsible for the entire SED, from IR up to $\mathrm{TeV}$ energies (see Fig. 3). As a consequence of the large compactness implied by the short variability timescale, the system naturally produces a powerful SSC component peaking in the X-ray band. As detailed in the Appendix A, the short variability timescale and the condition that the SSC component lies below the observed X-ray spectrum constrains the ratio $B_{\mathrm{b}} / \delta_{\mathrm{b}}^{5} \sim 10^{-9} \mathrm{G}$. Therefore, either we adopt a "standard" value of the magnetic field $\left(B_{\mathrm{b}}=0.1-1 \mathrm{G}\right)$ using extremely large Doppler factors, $\delta_{\mathrm{b}}>50$ or, conversely, we fix $\delta_{\mathrm{b}}$ to smaller values $\delta_{\mathrm{b}} \sim 10$ with extremely low magnetic fields, $B_{\mathrm{b}} \sim 10^{-4} \mathrm{G}$. If the blob is the result of reconnection events in the jet, which are expected to produce rather fast outflows (Giannios et al. 2009), we adopt in our model the first possibility, using $\delta_{\mathrm{b}}=75$.

As commonly found in FSRQs (e.g., the discussion in Celotti \& Ghisellini 2008), the condition that the low energy tail of the EC component reproduces the X-ray spectrum implies that the value of $\gamma_{\min }$ is constrained to be close to unity. As a consequence, the large number of particles at low energy pushes the total power demand of the region (calculated assuming one cold proton per electron) to extremely large values, close to $P_{\text {jet }}=3 \times 10^{47} \mathrm{erg} \mathrm{s}^{-1}$ (see Table 1). It would be possible to decrease the requested power allowing larger values of the Doppler factor $\delta_{\mathrm{b}}$. However, even with an extreme value of $\delta_{\mathrm{b}}=100$ the power remains quite large. Such large jet powers are not uncommon among FSRQs in bright states (e.g. Ghisellini et al. 2010; see also Bonnoli et al. 2011, for the specific case of 3C 454.3, which has a similar $L_{\mathrm{d}}$ ) and therefore we cannot exclude this possibility for PKS $1222+216$.

In Fig. 4, we present the results of the two-zone model in the two cases discussed above. In case B (left), we assume that both regions are cospatial and located outside the BLR, at distances $d>R_{\mathrm{BLR}}$; in case $\mathrm{C}$ (right), the large region is inside the BLR, while the blob is kept at the same distance as before. For the purposes of the calculation the exact distance of the two regions is not important, since both the radiation field of the BLR and the torus are uniform within the corresponding radii.

As demonstrated in Appendix B, in case (B) we find that the radiative coupling between the two regions is weaker than the EC flux using IR photons as seeds for the IC emission. Therefore, the EC/J and EC/b components can be safely neglected.

Since, in both cases, we are dealing with two separate regions, the number of parameters is relatively large, hence we have some freedom when selecting their values. However, the choice is not arbitrary: in the emission models shown in Fig. 4, we were guided by the following criteria.

Compact blob - We assume that it dominates the $\gamma$-ray emission but makes a small contribution to the X-ray band (but see below). This allows us to fix $\gamma_{\mathrm{p}}$, to put a lower limit on $\gamma_{\max }$, and to impose a lower limit on the minimum Lorentz factor of the emitting electrons (thus decreasing the total power request). As in case A (see discussion above), we assume a large bulk Lorentz factor to keep the SSC emission below the observed X-ray luminosity. Finally, the magnetic field and the electron density are determined by the level of the $\gamma$-ray emission and by the condition that the synchrotron emission lies below the observed optical flux level.

Large region - To constrain the properties of the IC component, we are guided by the X-ray spectrum and by the observed flux. Moreover, we assume that the component's $\mathrm{MeV}-\mathrm{GeV}$ emission contributes substantially to the total observed flux, although we assume that it lies a factor $\approx 2$ below the blob emission since the LAT and the MAGIC spectra suggest that they describe a unique component. The synchrotron peak is constrained by the 
Table 1. Input parameters for the emission models shown in Fig. 3 (first row) and Fig. 4 (other rows); "in" and "out" means inside or outside the BLR.

\begin{tabular}{lccccccccccccc}
\hline \hline & $\gamma_{\min }$ & $\gamma_{\mathrm{p}}$ & $\gamma_{\max }$ & $n_{1}$ & $n_{2}$ & $B[\mathrm{G}]$ & $K\left[\mathrm{~cm}^{-3}\right]$ & $R[\mathrm{~cm}]$ & $\delta$ & $\Gamma$ & $P_{\mathrm{p}, 45}$ & $P_{\mathrm{e}, 45}$ & $P_{\mathrm{B}, 45}$ \\
\hline All Blob & 3 & 1200 & $5 \times 10^{4}$ & 2.2 & 3.7 & 0.28 & $3 \times 10^{6}$ & $9.2 \times 10^{14}$ & 75 & 50 & 247 & 2.2 & $8.2 \times 10^{-4}$ \\
\hline Jet (in) & 1 & 300 & $6 \times 10^{4}$ & 2 & 3.45 & 0.75 & $1.8 \times 10^{4}$ & $1.5 \times 10^{16}$ & 19.7 & 10 & 81 & 0.34 & $6.3 \times 10^{-2}$ \\
Blob & 200 & 700 & $4 \times 10^{5}$ & 2.2 & 3.4 & 0.1 & $6 \times 10^{6}$ & $7.2 \times 10^{14}$ & 75 & 50 & 10.6 & 0.4 & $6.5 \times 10^{-5}$ \\
\hline Jet (out) & 3 & $2 \times 10^{3}$ & $6 \times 10^{4}$ & 2 & 4.1 & 0.09 & $3 \times 10^{2}$ & $1.1 \times 10^{17}$ & 19.7 & 10 & 17.7 & 0.3 & $5 \times 10^{-2}$ \\
Blob & 100 & 900 & $4 \times 10^{5}$ & 2.2 & 3.6 & 0.18 & $10^{7}$ & $6.2 \times 10^{14}$ & 75 & 50 & 3.9 & 0.8 & $1.5 \times 10^{-4}$ \\
\hline
\end{tabular}

Notes. The parameters are: the minimum $\left(\gamma_{\min }\right)$, break $\left(\gamma_{\mathrm{p}}\right)$ and maximum $\left(\gamma_{\max }\right)$ Lorentz factor and the low energy $\left(n_{1}\right)$ and the high energy $\left(n_{2}\right)$ slope of the smoothed power law electron energy distribution, the magnetic field $B$, the normalization of the electron distribution, $K$, the radius of the emission region, $R$, the Doppler factor $\delta$ and the corresponding bulk Lorentz factor $\Gamma$. Doppler factors are calculated assuming that the observer lies at an angle $\theta_{\mathrm{v}}=0.7^{\circ}$ from the jet axis. The last three columns report the power carried by (cold) protons (assuming one proton per emitting electron), relativistic electrons and magnetic field in units of $10^{45} \mathrm{erg} \mathrm{s}^{-1}$.

new IR data. For case C (emission inside the BLR), we tried to use values of the physical parameters commonly inferred in FSRQs (e.g. Ghisellini et al. 2009a,b).

In conclusion, while we have been unable to determine parameters uniquely, their values are reasonably constrained by the conditions posed by the SED and the observed variability. The parameters used for the SEDs shown in Figs. 3, 4 are reported in Table 1. Doppler factors are calculated assuming that the jet is observed at an angle $\theta_{v}=0.7^{\circ}$ from the axis. This relatively small angle is required because of the assumed large value of the Doppler factor of the blob, $\delta_{\mathrm{b}}=75$. The last three columns report the power carried by cold protons, relativistic electrons, and magnetic field. For the proton component, we assume one proton per relativistic electron. For cases $\mathrm{B}$ and $\mathrm{C}$, the power carried by the jet is in both cases exceedingly higher than that of the blob. This differs somewhat from similar models studied for the BL Lac PKS 2155-304 in Ghisellini \& Tavecchio (2008), where the power inferred for the blob was larger by more than one order of magnitude than that of the jet component. One reason for the difference is that the high external energy density makes the emission from the blob much more efficient than in the case of PKS 2155-304 (for which the dominant radiation field was that of the jet), thus reducing the required number of particles. Moreover, the total number of protons is also minimized by the fairly large minimum Lorentz factor of the emitting electrons. We also note that in all cases, for both the blob and the jet, the resulting intensity of the magnetic field is rather low. In turn this also implies that the associated Poynting flux is negligible compared to the power carried by the jet in the form of electrons and protons. The jet is therefore strongly matter dominated. This conclusion is robust, being the result of the very large observed ratio of the high energy (IC) to low energy (synchrotron) bumps.

\section{Discussion and conclusions}

It is puzzling to detect intense, hard, and rapidly variable VHE $\gamma$-ray emission from FSRQs, in addition to thermal emission from the accretion disk and broad emission lines. The observed very rapid variability constrains the size of the emitting region to be small, suggesting that its location in the jet is within the BLR. However, in this case the high energy photons interact with the emission line photons targets, and get absorbed.

To resolve this puzzle, we need to invoke a compact region located outside the BLR, whose size is smaller than the expected cross-sectional radius of the jet at that distance. This scenario follows similar ideas proposed to explain the ultra-fast VHE emission observed in the BL Lac objects PKS 2155-304 and Mkn 501. However, the case of FSRQs provides a tighter constraint, because, to avoid the strong absorption by the BLR photons, the blob must be located at large distances, $\gtrsim 0.1 \mathrm{pc}$. In BL Lacs, the relatively "clean" environment is instead rather transparent to $\gamma$-ray photons (at least up to the $\mathrm{TeV}$ band, above which the thermal radiation from a dusty torus could affect the emission, e.g. Celotti et al. 1998) and the emission region could reside much closer to the central engine.

As for BL Lacs, in FSRQs such compact blobs could originate from reconnection events that could naturally produce compact regions of rapidly moving plasma inside the jet (the so-called "jet in a jet" scenario; Giannios et al. 2009, 2010; Nalewajko et al. 2011). However, with respect to the case of BL Lac objects, in which the emission and the electron cooling are dominated by the SSC process, the denser external radiation field is expected to dominate the radiation energy density, leading to stronger radiative losses, lower average particle energies, and, consequently, an emission peaking at $\mathrm{GeV}$ energies. The total derived power carried by the "blob" is much less than the total jet power, supporting the energetic sustainability of the process, and the possibility that there are, at any given time, several active blobs, pointing in slightly different directions. This would increase the otherwise small probability of having very small viewing angles (see also discussions in Ghisellini et al. 2009a,b; and Giannios et al. 2010). It is conceivable that the events leading to the production of these fast blobs can occur also at smaller distances from the central $\mathrm{BH}$, even within the BLR. In this case, the dense radiation field would prevent the escape of the VHE photons and the entire power emitted above few tens of $\mathrm{GeV}$ would be eventually reprocessed at lower energies. A non-negligible magnetic field within the BLR would spread the directions of the emitting pairs into a broad cone, thus decreasing the observed flux and the chances of detection of this reprocessed radiation. Although this scenario is attractive, it is based on the assumption that the plasma in the emission region is magnetically dominated, with a magnetization parameter (i.e. Poynting to kinetic flux ratio) of about 10-100. In contrast, our models show that the flow at the distances where radiation is produced is characterized by a rather low value of the magnetic flux compared to that carried by electrons or protons.

The severe radiative losses of the electrons in the disk and BLR radiation fields seem to also rule out the alternative possibility that the rapidly varying VHE emission originates in extremely collimated beams of ultra-relativistic electron, as envisaged in the "needle" scenario of Ghisellini et al. (2009a,b). Owing to the strong dependence of the maximum electron Lorentz factor (resulting from the competition between the 
magneto-centrifugal acceleration in the black hole magnetosphere - e.g., Rieger \& Mannheim 2000 - and the strong IC cooling) on the radiation energy density, $\gamma_{\max } \propto U_{\text {rad }}^{-2}$ (Osmanov et al. 2007), the presence of a luminous disk and BLR will result in rather low energy of the electrons, emitting at energies below the GeV band.

Another possible explanation of a very small region at large distance is the focusing of the jet by means of strong recollimation (e.g. Komissarov \& Falle 1997; Sokolov et al. 2004; Stawarz et al. 2006; Nalewajko \& Sikora 2008; Bromberg \& Levinson 2009). In particular, Bromberg \& Levinson (2009) showed that if the shock resulting from the recollimation of the jet by the interaction with an external medium is efficient in converting the dissipated energy into radiation, the reduced post-shock pressure allows the jet to be strongly "squeezed", reaching a minimum size close to the point where the recollimation shock crosses the jet axis. At this point, reflection (internal) shocks are expected to form (e.g., Komissarov \& Falle 1997), and the shortest variability time should be constrained by the typical size of these structures, which is of the order of the radius of the "nozzle" of the jet. Under the assumption that the external confining medium is associated with matter in the BLR, Bromberg \& Levinson (2009) estimate that the re-collimation shock reaches the jet axis at a distance $d^{\star} \simeq 2.5 L_{\mathrm{j}, 46}\left(R_{\mathrm{BLR}} / 0.1 \mathrm{pc}\right)^{-1}$ pc where $L_{\mathrm{j}}$ is the jet power. Their simulations show that at this point if $(i)$ the cooling operates at a rate higher than that of the dynamical timescale and (ii) the radiative conversion efficiency is sufficiently large (30 percent of the bulk luminosity is converted into radiation), the cross-sectional radius of the jet in the focusing point can be as small as $R \sim 10^{-2.5} d^{\star}$. Condition $(i)$ is easily fulfilled in FSRQs, even outside the BLR. Nalewajko \& Sikora (2009) showed that the efficiency of internal energy production in recollimation shocks can be larger than $30 \%$ provided that the product of the jet bulk Lorentz factor and the aperture angle is larger than $\approx 3$. Therefore, provided that the conversion of this dissipated energy into radiation occures with large efficiencies, condition (ii) could also be satisfied. Owing to the rough consistency of these numbers with the results of our radiative models and the difficulties faced by the "jet in a jet" or "needles" models discussed above, we believe that such a scenario is rather promising and worthy of closer investigation.

Acknowledgements. We are grateful to an anonymous referee for constructive comments that helped us to substantially improve the paper. We thank Amir Levinson for discussions.

\section{Appendix A: Limits to $B$ and $\delta$ from the constraints on the SSC component}

One can derive an approximate relation between the magnetic field and the Doppler factor (assuming for simplicity $\delta_{\mathrm{b}}=\Gamma_{\mathrm{b}}$ ) starting from the approximate expressions for the EC and SSC luminosities

$$
L_{\mathrm{SSC}}=\frac{4}{3} \sigma_{\mathrm{T}} c U_{\mathrm{synch}}^{\prime}\left\langle\gamma^{2}\right\rangle n V \delta_{\mathrm{b}}^{4}
$$

and

$$
L_{\mathrm{EC}}=\frac{4}{3} \sigma_{\mathrm{T}} c U_{\mathrm{ext}} \Gamma_{\mathrm{b}}^{2}\left\langle\gamma^{2}\right\rangle n V \delta_{\mathrm{b}}^{4}
$$

where $U_{\text {synch }}^{\prime}$ and $U_{\text {ext }} \Gamma_{\mathrm{b}}^{2}$ are the energy density of the synchrotron and external radiation in the blob frame, $V=4 / 3 \pi R_{\mathrm{b}}^{3}$ is the blob volume, $n$ is the electron density, and $\left\langle\gamma^{2}\right\rangle$ is the average value of the square of the electron Lorentz factor.
Using these equations, and assuming that that $L_{\mathrm{EC}}=L_{\gamma}$, $L_{\mathrm{SSC}} / L_{\mathrm{EC}}<L_{\mathrm{X}} / L_{\gamma}$, and $R_{\mathrm{b}} \simeq c t_{\mathrm{var}} \delta_{\mathrm{b}}$, with some manipulations, one can derive a relation between the magnetic field and the Doppler factor (assuming for simplicity $\delta_{\mathrm{b}}=\Gamma_{\mathrm{b}}$ ) containing only observed quantities

$B_{\mathrm{b}} \lesssim\left[\frac{32 \pi^{2} c^{3}}{L_{\gamma}}\left(\frac{L_{\mathrm{X}}}{L_{\gamma}}\right)\right]^{1 / 2} U_{\mathrm{ext}} t_{\mathrm{var}} \delta_{\mathrm{b}}^{5}$.

In our specific case where $t_{\mathrm{var}}=600 \mathrm{~s}, L_{\gamma} \simeq 3 \times 10^{47} \mathrm{erg} \mathrm{s}^{-1}$, $L_{\mathrm{X}} / L_{\gamma} \lesssim 10^{-3}$, and $U_{\text {ext }} \equiv U_{\mathrm{IR}}=5 \times 10^{-4} \mathrm{erg} \mathrm{cm}^{-3}$, we derive $B_{\mathrm{b}} \simeq 10^{-4}\left(\delta_{\mathrm{b}} / 10\right)^{5} \mathrm{G}$.

\section{Appendix B: Importance of the blob/jet radiative interplay}

The ratio of the EC/IR to EC/J (inverse Compton produced in the blob scattering photons of the jet) luminosities is given by the ratio of the energy densities of the corresponding seed photons in the blob rest-frame

$\frac{L_{\mathrm{EC} / \mathrm{IR}}}{L_{\mathrm{EC} / \mathrm{J}}}=\frac{U_{\mathrm{IR}}^{\prime}}{U_{\mathrm{J}}^{\prime}}$

where the primed quantities are measured in the blob rest-frame. The two energy densities can be expressed as

$U_{\mathrm{IR}}^{\prime}=\frac{L_{\mathrm{IR}} \Gamma_{\mathrm{b}}^{2}}{4 \pi R_{\mathrm{IR}}^{2} c}, \quad U_{\mathrm{J}}^{\prime}=\frac{L_{\mathrm{J}} \Gamma_{\mathrm{rel}}^{2}}{4 \pi R_{\mathrm{J}}^{2} \delta_{\mathrm{J}}^{4} c}$,

where "b" and "J" refers to quantities related to the blob and the jet, respectively, and $\Gamma_{\text {rel }}=\Gamma_{\mathrm{b}} \Gamma_{\mathrm{J}}\left(1-\beta_{\mathrm{b}} \beta_{\mathrm{J}}\right)$ is the relative bulk Lorentz factor between the jet flow and the blob. Therefore we finally find that

$\frac{L_{\mathrm{EC} / \mathrm{IR}}}{L_{\mathrm{EC} / \mathrm{J}}}=\frac{L_{\mathrm{IR}}}{L_{\mathrm{J}}}\left(\frac{R_{\mathrm{J}}}{R_{\mathrm{IR}}}\right)^{2}\left(\frac{\Gamma_{\mathrm{b}}}{\Gamma_{\text {rel }}}\right)^{2} \delta_{\mathrm{J}}^{4}$.

Since $L_{\mathrm{IR}}$ is comparable to the observed $L_{\mathrm{J}}$ (see Fig. 1), $R_{\mathrm{IR}} \sim$ $10 \times R_{\mathrm{J}}, \Gamma_{\mathrm{b}}=50, \Gamma_{\mathrm{J}}=10$, and $\delta_{\mathrm{J}} \sim 20$, Eq. (B.3) implies that the EC/IR component largely dominates over the EC/J component, $L_{\mathrm{EC} / \mathrm{IR}} / L_{\mathrm{EC} / \mathrm{J}} \approx 10^{6}$.

Analogously, one can evaluate the ratio of the EC/IR to EC/b (inverse Compton produced in the jet scattering photons of the blob) luminosities

$\frac{L_{\mathrm{EC} / \mathrm{IR}}}{L_{\mathrm{EC} / \mathrm{b}}}=\frac{U_{\mathrm{IR}}^{\prime}}{U_{\mathrm{b}}^{\prime}}$,

where primed quantities are now measured in the jet frame. As above, this expression can be rewritten as:

$\frac{L_{\mathrm{EC} / \mathrm{IR}}}{L_{\mathrm{EC} / \mathrm{b}}}=\frac{L_{\mathrm{IR}}}{L_{\mathrm{b}}}\left(\frac{R_{\mathrm{J}}}{R_{\mathrm{IR}}}\right)^{2}\left(\frac{\delta_{\mathrm{b}}}{\delta_{\mathrm{rel}}}\right)^{4} \frac{1}{\Gamma_{\mathrm{J}}^{2}}$.

Again, since $L_{\mathrm{IR}}$ is comparable to or higher than the observed $L_{\mathrm{B}}, R_{\mathrm{IR}} \sim 10 \times R_{\mathrm{J}}, \Gamma_{\mathrm{b}}=50, \Gamma_{\mathrm{J}}=10$, and $\delta_{\mathrm{J}} \sim 20$, we find a high ratio $L_{\mathrm{EC} / \mathrm{IR}} / L_{\mathrm{EC} / \mathrm{b}} \gg 1$.

Therefore, both the EC/J and EC/b components can be safely neglected. 


\section{References}

Abdo, A. A., Ackermann, M., Ajello, M., et al. 2010, ApJ, 722, 520

Abdo, A. A., Ackermann, M., Ajello, M., et al. 2011, Science, 331, 739

Aharonian, F., Akhperjanian, A. G., Bazer-Bachi, A. R., et al. 2007, ApJ, 664, L71

Albert, J., Aliu, E., Anderhub, H., et al. 2007, ApJ, 669, 862

Albert, J., Aliu, E., Anderhub, H., et al. 2008, Science, 320, 1752

Aleksić, J., Antonelli, L. A., Antoranz, P., et al. 2011a, A\&A, 530, 4

Aleksić, J., Antonelli, L. A., Antoranz, P., et al. 2011b, ApJ, 730, L8

Aloy, M. A., Janka, H.-T., \& Mueller, E. 2005, A\&A, 436, 273

Bednarek, W., \& Idec, W. 2011, MNRAS, 551

Bentz, M. C., Walsh, J. L., Barth, A. J., et al. 2009, ApJ, 705, 199

Błażejowski, M., Sikora, M., Moderski, R., \& Madejski, G. M. 2000, ApJ, 545, 107

Bloom, S. D., \& Marscher, A. P. 1996, ApJ, 461, 657

Bonnoli, G., Ghisellini, G., Foschini, L., Tavecchio, F., \& Ghirlanda, G. 2011, MNRAS, 410, 368

Bromberg, O., \& Levinson, A. 2009, ApJ, 699, 1274

Celotti, A., \& Ghisellini, G. 2008, MNRAS, 385, 283

Celotti, A., Fabian, A. C., \& Rees, M. J. 1998, MNRAS, 293, 239

Chonis, T. S., \& Gaskell, C. M. 2008, AJ, 135, 264

de Angelis, A., Mansutti, O., \& Persic, M. 2008, Nuovo Cimento, 31, 187

Decarli, R., Dotti, M., \& Treves, A. 2011, MNRAS, 413, 39

Dermer, C. D., Finke, J. D., Krug, H., \& Böttcher, M. 2009, ApJ, 692, 32

Domínguez, A., Primack, J. R., Rosario, D. J., et al. 2011, MNRAS, 410, 2556

Fan, Z., Cao, X., \& Gu, M. 2006, ApJ, 646, 8

Foschini, L., Ghisellini, G., Tavecchio, F., Bonnoli, G., \& Stamerra, A. 2011, A\&A, 530, 77

Ghisellini, G., \& Tavecchio, F. 2008, MNRAS, 386, L28

Ghisellini, G., \& Tavecchio, F. 2009, MNRAS, 397, 985

Ghisellini, G., Celotti, A., Fossati, G., Maraschi, L., \& Comastri, A. 1998, MNRAS, 301, 451

Ghisellini, G., Tavecchio, F., Bodo, G., \& Celotti, A. 2009a, MNRAS, 393, L16

Ghisellini, G., Foschini, L., Volonteri, M., et al. 2009b, MNRAS, 399, L24

Ghisellini, G., Tagliaferri, G., Foschini, L., et al. 2011, MNRAS, 411, 901

Giannios, D., Uzdensky, D. A., \& Begelman, M. C. 2009, MNRAS, 395, L29

Giannios, D., Uzdensky, D. A., \& Begelman, M. C. 2010, MNRAS, 402, 1649

Liu, H. T., \& Bai, J. M. 2006, ApJ, 653, 1089

Hinton, J. A., \& Hofmann, W. 2009, ARA\&A, 47, 523

Jarvis, M. J., \& McLure, R. J. 2006, MNRAS, 369, 182
Kaspi, S., Brandt, W. N., Maoz, D., et al. 2007, ApJ, 659, 997

Komissarov, S. S., \& Falle, S. A. E. G. 1997, MNRAS, 288, 833

Komissarov, S. S., \& Lyutikov, M. 2011, MNRAS, 518

Kubo, H., Takahashi, T., Madejski, G., et al. 1998, ApJ, 504, 693

Malmrose, M., Marscher, A., Jorstad, S., Nikutta, R., \& Elitzur, M. 2011, ApJ, 732,116

Maraschi, L., \& Tavecchio, F. 2003, ApJ, 593, 667

Maraschi, L., Ghisellini, G., \& Celotti, A. 1992, ApJ, 397, L5

Marscher, A. P., \& Jorstad, S. G. 2010, in Proc. Fermi Meets Jansky [arXiv: 1005.5551$]$

Marscher, A. P., Jorstad, S. G., D’ Arcangelo, F. D., et al. 2008, Nature, 452, 966 Nalewajko, K., \& Sikora, M. 2009, MNRAS, 392, 1205

Nalewajko, K., Giannios, D., Begelman, M. C., Uzdensky, D. A., \& Sikora, M. 2011, MNRAS, 413, 333

Osmanov, Z., Rogava, A., \& Bodo, G. 2007, A\&A, 470, 395

Osterbrock, D. E., \& Pogge, R. W. 1987, ApJ, 323, 108

Padovani, P., \& Giommi, P. 1995, ApJ, 444, 567

Poutanen, J., \& Stern, B. 2010, ApJ, 717, L118

Rieger, F. M., \& Mannheim, K. 2000, A\&A, 353, 473

Shields, G. A. 1978, in Proc. of the Pittsburgh Conf. on BL Lac objects, ed. A. M. Wolfe, 275

Sikora, M., Begelman, M. C., \& Rees, M. J. 1994, ApJ, 421, 153

Sikora, M., Moderski, R., \& Madejski, G. M. 2008, ApJ, 675, 71

Sokolov, A., Marscher, A. P., \& McHardy, I. M. 2004, ApJ, 613, 725

Spada, M., Ghisellini, G., Lazzati, D., \& Celotti, A. 2001, MNRAS, 325, 1559

Stawarz, Ł., Aharonian, F., Kataoka, J., et al. 2006, MNRAS, 370, 981

Stern, B. E., \& Poutanen, J. 2011, MNRAS, in press [arXiv: 1105.2762]

Tagliaferri, G., et al. 2008, ApJ, 679, 1029

Tanaka, Y. T., Stawarz, L., Thompson, D. J., et al. 2011, ApJ, 733, 19

Tavani, M., Bulgarelli, A., Vittorini, V., et al. 2011, Science, 331, 736

Tavecchio, F., \& Ghisellini, G. 2008, MNRAS, 386, 945

Tavecchio, F., \& Mazin, D. 2009, MNRAS, 392, L40

Tavecchio, F., Maraschi, L., \& Ghisellini, G. 1998, ApJ, 509, 608

Tavecchio, F., Maraschi, L., Ghisellini, G., et al. 2000, ApJ, 543, 535

Tavecchio, F., Maraschi, L., Ghisellini, G., et al. 2002, ApJ, 575, 137

Tavecchio, F., Ghisellini, G., Bonnoli, G., \& Ghirlanda, G. 2010, MNRAS, 405, L94

Tornikoski, M., Valtaoja, E., Teraesranta, H., et al. 1996, A\&AS, 116, 157

Vittorini, V., Tavani, M., Pucella, G., et al. 2011, ApJ, 732, L22

Wagner, S., \& Behera, B. 2010, in 10th HEAD Meeting, Hawaii, BAAS, 42, 2 , 07.05 